\title{
APPROXIMATE EVALUATION OF INTEGRALS
}

\author{
ROGER J. HOSKING ${ }^{1}$
}

(Received 16 March 1998)

\begin{abstract}
Asymptotic and numerical analysis provides essential insight into the behaviour of Fourier integral solutions for the deflexion of an infinite continuously-supported flexible plate due to moving load. Thus we can define in detail how the plate defiexion depends upon the load speed, including (a) the wave patterns generated by a load moving steadily at various supercritical speeds; and (b) the time-dependent behaviour of the deflexion due to an impulsively-started load, where the two-dimensional response tends to a steady state except at the critical speed, when it grows continuously with time (in the absence of dissipation).
\end{abstract}

\section{Introduction}

The approximate evaluation of integrals is not only a recognised area of numerical analysis but also a major branch of asymptotic analysis, which can often provide valuable insight into the behaviour of solutions to problems in the mathematical sciences. An asymptotic solution may be sought when an exact mathematical solution or a numerical solution is difficult to interpret or even obtain, and asymptotic analysis in combination with numerical computation can also produce important results. It is a great pleasure for me to participate in this festschrift for Professor David Elliott to celebrate his 65th birthday, and I hope my small part at the associated meeting contributed to that clear demonstration of the high regard in which he is held by the mathematical community.

The mathematical theory for the response of a flexible continuously-supported beam or plate to a moving load is a case example of the application of asymptotic analysis and numerical computation. ${ }^{2} \mathrm{~A}$ combination of asymptotic analysis and numerical computation yields the steady-state wave patterns generated in the plate by a

\footnotetext{
'Department of Mathematics, Universiti Brunei Darussalam, Gadong BE1410, Brunei.

(C) Australian Mathematical Society 2000, Serial-fee code 0334-2700/00

2 The beam or plate may represent a rail or road surface, an airport runway or a floating ice sheet in a cold region. The moving load might be a conventional vehicle, a landing aeroplane or a hovercraft. Moving Loads on Ice Plates is the title of a recent monograph [10], where it is emphasised that the
} 
steadily-moving load ([1]). Secondly, asymptotic analysis supplemented by numerical computation renders the time-dependent response $([8,11]$ and $[7])$.

\section{Modelling moving loads over flexible continuously-supported plates}

The simplest accepted mathematical model to describe the response of a continuously-supported plate to a moving load corresponds to a thin plate of infinite extent resting on a foundation of some kind. Thus we assume that the vertical deflexion of the plate $\zeta(x, y, t)$ satisfies the partial differential equation

$$
D \nabla^{4} \zeta+\rho^{\prime} h \frac{\partial^{2} \zeta}{\partial t^{2}}=p-f(x, y, t)
$$

or its visco-elastic counterpart [10]. Here $\nabla^{2}=\partial^{2} / \partial x^{2}+\partial^{2} / \partial y^{2}$ is the usual Laplacian differential operator, where $(x, y)$ denotes any field point in the plane of the plate, and $t$ is the time; $D$ is the effective flexural rigidity of the plate; $\rho^{\prime}$ and $h$ are the plate density and thickness respectively; $p$ is the pressure exerted by the underlying foundation on the plate; and $f(x, y, t)$ is the downward forcing function on the plate due to the moving load (see below).

In the context of a floating ice plate, as in all the research papers listed in the previous section, the motion of the underlying foundation (water) is defined by a velocity potential $\phi(x, y, z, t)$. Thus from Bernoulli's equation for incompressible irrotational flow we have the pressure at the plate

$$
p=-\rho\left(\frac{\partial \phi}{\partial t}\right)_{z=0}-\rho g \zeta,
$$

where $\rho$ is the water density and $g$ is the gravitational acceleration. We then express $p$ entirely in terms of $\zeta$, using the kinematic non-cavitation boundary condition

$$
\frac{\partial \zeta}{\partial t}=\left(\frac{\partial \phi}{\partial z}\right)_{z=0}
$$

and invoke the Fourier transform

$$
\hat{\zeta}\left(k_{1}, k_{2}, \omega\right)=(2 \pi)^{-3 / 2} \int \zeta(x, y, t) e^{i\left(k_{1} x+k_{2} y-\omega t\right)} d x d y d t
$$

to formally solve (1) for the deflexion of the infinite plate.

deflexion can be much greater when the load is moving than when it is stationary. Hovercraft exploiting this resonance phenomenon are used as ice breakers on the St. Lawrence Seaway in Canada, but the safe operation of transport systems of course usually means avoiding any fracture! 


\section{Steadily moving loads}

For a steady two-dimensional load moving in the $x$-direction with uniform speed $V$, on inverting (2) we obtain the solution as the Fourier integral

$$
\zeta(x, y, t)=-\frac{1}{2 \pi} \int \frac{\hat{F}\left(k_{1}, k_{2}\right)}{B\left(k_{1}, k_{2}\right)} e^{-i\left[k_{1}(x-v t)+k_{2} y\right]} d k_{1} d k_{2},
$$

since the Fourier transform of the loading function $f(x, y, t)=F(x-V t, y)$ is $\hat{f}\left(k_{1}, k_{2}, \omega\right)=\sqrt{2 \pi} \delta\left(V k_{1}-\omega\right) \hat{F}\left(k_{1}, k_{2}\right)$, and we write $B\left(k_{1}, k_{2}\right)=D k^{4}+\rho g-$ $\rho h^{\prime} V^{2} k_{1}^{2}-\left(\rho V^{2} k_{1}^{2} / k\right) \operatorname{coth}(k H)$, where wavenumber $k=\sqrt{k_{1}^{2}+k_{2}^{2}}$ and $h^{\prime}=\rho^{\prime} h / \rho$. There are hybrid (flexural-gravity) waves generated in the plate by the moving load when the load speed is supercritical - that is, for $V>c_{\min }$, where $c_{\min }$ is the minimum phase speed of these waves. (At subcritical load speeds $V<c_{\min }$, the deflexion given by (3) closely resembles that produced when the load is stationary.) Note that $B\left(k_{1}, k_{2}\right)=0$ is just the dispersion relation with $\omega$ replaced by $V k_{1}$ as required for a steady wave pattern, since the component of the load velocity normal to any wave crest $\left(V k_{1} / k\right)$ must equal the crest phase speed $(\omega / k)$. We can not expect to evaluate such an integral exactly, but we can exploit asymptotic methods and numerical computation.

It is convenient to choose the observation time to be the instant the travelling load coincides with the origin of the $(x, y)$-plane; and from the Cauchy residue theorem we can use contour integration with respect to $k_{1}$ to first obtain

$$
\zeta(x, y)=i \int_{C_{k}} \frac{\hat{F}\left(k_{1}, k_{2}\right)}{B_{k_{1}}\left(k_{1}, k_{2}\right)} e^{-i\left(k_{1} x+k_{2} y\right)} d k_{2},
$$

due to the pole at $k_{1}\left(k_{2}\right)$ associated with the point on the wavenumber curve $C_{k}$ defined in the real $\left(k_{1}, k_{2}\right)$-plane by $B\left(k_{1}, k_{2}\right)=0$ (see [4]). It turns out that we can produce useful formulae for the deflexion at distances somewhat larger than the load dimension by asymptotic analysis, which at the same time allows us to treat the load as a concentrated point source.

For most directions away from the load, we get the stationary phase approximation

$$
\zeta(x, y) \sim-F\left(k_{10}, k_{20}\right)\left[\left(\frac{\partial B}{\partial n}\right)_{0}\right]^{-1} \sqrt{\frac{2 \pi}{\kappa_{0} r}} e^{-i\left(k_{0} . x+\Theta\right)}
$$

as $r=\sqrt{x^{2}+y^{2}} \rightarrow \infty$, where $\kappa_{0} \equiv\left|k_{1}^{\prime \prime}\left(k_{20}\right)\right|$ denotes the small curvature on $C_{k}$ at the point $\left(k_{10}, k_{20}\right), \partial / \partial n$ denotes differentiation normal to $C_{k}$ in the sense of increasing $\omega$, and $\Theta$ is a phase factor equalling $\pi / 4$ if $C_{k}$ is convex to the $n$-direction at the point $\left(k_{10}, k_{20}\right)$ and $\pi$ otherwise ([1]). At sufficiently high supercritical load speeds $V$, there are variations due to the emergence of caustics associated with points of inflexion on $C_{k}$ 
(where $k_{1}^{\prime}\left(k_{2}\right)=k_{1}^{\prime \prime}\left(k_{2}\right)=0$ ), and indeed also "supercaustics" associated with points on $C_{k}$ where two caustics just merge (where $k_{1}^{\prime}\left(k_{2}\right)=k_{1}^{\prime \prime}\left(k_{2}\right)=k_{1}^{\prime \prime \prime}\left(k_{2}\right)=0$ ). All of this enables us to ascertain preferred directions of energy propagation corresponding to caustics or supercaustics, and to compute the wave patterns that are notably dependent on the supercritical load speed ([1]). Incidentally, a "shadow zone" appears behind the load when the load speed $V$ exceeds the water wave speed $\sqrt{g H}$.

Milinazzo et al. [5] numerically evaluated the integral (4) for a distributed (rectangular) load, to produce quite remarkable analogous wave pattern plots. They noted that their computed steady state solution appears bounded when $V=\sqrt{g H}$, and indeed at all (subcritical or supercritical) load speeds considered except the critical speed $V=c_{\min }$. Any large resonant response at some particular load speed is obviously a matter of considerable practical importance ( $c f$. the footnote on page 110), but successive mathematical investigation of this phenomenon in our context of moving loads over floating flexible plates has produced an amusing litany of conflicting predictions.

The pioneering paper by Kheysin [2] discussed the deflexion due to either a steadilymoving concentrated point load or a steadily-moving line load on a floating flexible plate (as modelled in Section 2), on the implicit assumption that a steady state exists. In the case of the concentrated point load, he erroneously concluded that there is no critical speed (at which the deflexion is unbounded); but for the one-dimensional response due to a line load, he correctly identified two such critical load speeds. Nevel [6] subsequently showed that there is a critical load speed for a steadilymoving uniformly distributed circular load at which the deflexion is unbounded. Further mathematical investigations of this critical speed phenomenon, but taking time-dependence into account, are described in the following section.

\section{Impulsively-started loads}

Kheysin [3] was the first to recognise that time-dependence might help explain the singularity of the steady-state deflexion due to a load moving steadily at a critical speed, since any steady-state response corresponds to the load acting for an infinite period of time. For an impulsively-started line load, he found a critical speed at which the deflexion $\zeta$ increases with time $t$ (as $t^{1 / 2}$ when $t \rightarrow \infty$ ). In their much later but more complete analysis for an impulsively-started line load, inter alia Schulkes and Sneyd [8] identified two load speeds at which the deflexion in the vicinity of the load increases with time $t$-viz. as $t^{1 / 2}$ when $V=c_{\min }$ and as $t^{1 / 3}$ when $V=\sqrt{g H}$.

However, when Milinazzo et al. [5] observed that their computed steady state deflexion due to a steadily-moving distributed (rectangular) load appeared to be finite at the load speed $V=\sqrt{g H}$, it became evident that time-dependent analysis for $a$ two-dimensional response was required ([10]). Indeed, in respect of one of the two 
speeds at which they had found the the deflexion does not tend to a steady state, Schulkes and Sneyd ([8]) noted it is "possible that $V=\sqrt{g H}$ does not represent a critical speed for two-dimensional sources, because wave energy could radiate in all directions-not just along the line of motion". Load concentration did not seem to be a factor however, since the linearity of the mathematical problem evidently permits generalisation of the response due to a point load to that due to a distributed load (by superposition), but this aspect might also be explored. Thus we were led to consider the time-dependent two-dimensional response due to a concentrated point load, and then the time-dependent response due to a distributed load.

For an impulsively-started concentrated point load, which subsequently travels with uniform speed $V$ along the $x$-axis, the appropriate loading function is

$$
f(x, y, t)=P_{0} \delta(x-V t) \delta(y) U(t),
$$

where $U(t)$ is the Heaviside unit step function and $P_{0}$ is constant. The corresponding deflexion is given by the Fourier double integral ([7])

$$
\begin{aligned}
\zeta(x, y, t)=- & \frac{P_{0}}{8 \pi^{2} \rho} \int_{-\infty}^{\infty} \int_{-\infty}^{\infty} \frac{\tanh (k H)}{c(k)} e^{-i\left[k_{1}(x-V t)+k_{2} y\right]} \\
& \times\left\{\frac{1-e^{i \Psi_{1} t}}{\Psi_{1}}+\frac{1-e^{-i \Psi_{2} t}}{\Psi_{2}}\right\} d k_{1} d k_{2},
\end{aligned}
$$

involving the phase functions $\Psi_{1}\left(k_{1}, k_{2}\right)=k c-k_{1} V$ and $\Psi_{2}\left(k_{1}, k_{2}\right)=k c+k_{1} V$ (where $k=\sqrt{k_{1}^{2}+k_{2}^{2}}$ as before). The phase speed $c(k)=\omega(k) / k$ is the positive root of the dispersion relation

$$
c^{2}=\left(\frac{D k^{4}}{\rho}+g\right) \frac{\tanh (k H)}{k}
$$

for real $k$. This result (5) is the two-dimensional generalisation of the one-dimensional Cartesian form for a line load, analysed by Kheysin [3] and Schulkes and Sneyd [8]. Thus to get the asymptotic behaviour for large time $t$, we might likewise consider only the term involving $\Psi_{1}$, since the other phase function $\Psi_{2}$ is monotonically increasing with $k$.

Using the polar coordinate transformation $x-V t=r \cos \xi, y=r \sin \xi, k_{1}=$ $k \cos \theta, k_{2}=k \sin \theta$, we can re-express (5) as

$$
\begin{aligned}
\zeta(r, \xi, t)=- & \frac{P_{0}}{4 \pi^{2} \rho} \operatorname{Re}\left(\int_{0}^{\infty} \int_{-\pi / 2}^{\pi / 2} \frac{\tanh (k H)}{c(k)} e^{-i k r \cos (\theta-\xi)}\right. \\
& \left.\times\left\{\frac{1-e^{i k(c-V \cos \theta) t}}{c-V \cos \theta}+\frac{1-e^{-i k(c+V \cos \theta) t}}{c+V \cos \theta}\right\} d \theta d k\right),
\end{aligned}
$$


where the polar coordinates $(r, \xi)$ define the field position on the plate in any direction relative to the moving load, and $\operatorname{Re}(z)$ denotes the real part of $z$.

To examine the evolution of the deflexion, from the asymptotic viewpoint we might consider either (5) or (6) in the limit as time $t \rightarrow \infty$, especially at the two load speeds $V=\sqrt{g H}$ and $V=c_{\min }$ where the one-dimensional response due to a line load was found to grow ([8]). As discussed at the meeting in honour of David Elliott however, it is at precisely each of these two load speeds that the stationary point of the important phase function $\Psi_{1}$ coincides with the pole of the associated amplitude function in the double integral of (5) or (6), which poses an interesting problem for asymptotic analysis. Thus in the polar coordinate form (6) for example, at these two load speeds the critical points (points of stationary phase) occur where $\theta=0$ and respectively (i) $k=0$ (when $V=\sqrt{g H}$ ) and (ii) $k=k_{\min }$ (when $V=c_{\min }$ )- that is, at the two load speeds when $V=c_{g}=c$, where $c_{g}=d \omega / d k$ denotes the group speed. We have since calculated the deflexion numerically from the complete form (5), because the zeros of the phase functions are zeros of the respective complete numerators, and enhanced the following asymptotic analysis due to Wang and Hosking ([11]) that was discussed at the meeting (see [7]).

An alternative expression for the polar coordinate form of the deflexion (6) is obtained by first representing the time behaviour as an integral, and then (more importantly) integrating with respect to $\theta$ to get

$$
\zeta(r, \xi, t)=-\frac{P_{0}}{2 \pi \rho} \int_{0}^{\infty} \frac{k \tanh (k H)}{c(k)} \int_{0}^{t} J_{0}(k A) \sin (k c s) d s d k,
$$

where $A=\sqrt{(r \cos \xi+V s)^{2}+r^{2} \sin ^{2}(\xi)}$. As discussed at the meeting, we can also generalize the idea of Nevel [6] to obtain the related time-dependent deflexion under the centre of a load uniformly distributed over a circular area of radius $R$. Thus we introduce

$$
\zeta(t)=\int_{0}^{R} \int_{0}^{2 \pi} \frac{\zeta(r, \xi, t)}{\pi R^{2}} r d r d \xi
$$

to get the result for a distributed load analogous to (7):

$$
\zeta(t)=-\frac{P_{0}}{\pi \rho R} \int_{0}^{\infty} \frac{\tanh (k H) J_{1}(k R)}{c(k)} \int_{0}^{t} J_{0}(k V s) \sin (k c s) d s d k .
$$

The eventual evolved (potentially steady-state) solution corresponds to setting $t=\infty$ in (7) or (8)-for example, the fully evolved deflexion under the centre of the circular load is

$$
\zeta_{\infty}=-\frac{P_{0}}{\pi \rho R} \int_{0}^{\infty} \frac{\tanh (k H) J_{1}(k R)}{c(k)} \int_{0}^{\infty} J_{0}(k V s) \sin (k c s) d s d k
$$




$$
=-\frac{P_{0}}{\pi \rho R} \int_{0}^{\infty} \frac{\tanh (k H) J_{1}(k R)}{c(k)} \frac{U(c-V)}{\sqrt{k^{2}\left(c^{2}-V^{2}\right)}} d k
$$

and on introducing $a=k c^{2} /[g \tanh (k H)]$ and $b=k V^{2} /[g \tanh (k H)]$ we obtain the integral derived by Nevel [6]:

$$
\begin{aligned}
\zeta_{\infty} & =-\frac{P_{0}}{\pi \rho R} \int_{0}^{\infty} \frac{J_{1}(k R)}{R} \frac{U(a-b)}{\sqrt{a(a-b)}} d k \\
& =-\frac{P_{0}}{\pi \rho g \ell^{2}} \int_{0}^{\infty} \frac{J_{1}(\xi R / \ell)}{R / \ell} \frac{U(a-b)}{\sqrt{a(a-b)}} d \xi,
\end{aligned}
$$

where $U$ is the Heaviside unit step function introduced earlier and $\xi=k \ell$ is the dimensionless wave number (where $\ell=(D / \rho g)^{1 / 4}$ is a characteristic length). Nonzero contributions to the integral correspond to wave numbers $k$ where $V<c(k)$, or equivalently $a>b$. Nevel noted that his integral solution (9) is unbounded when $a=b$ and $d a / d \xi=d b / d \xi$, which in the deep water limit where $\tanh (k H) \approx 1$ corresponds to

$$
V \approx 2\left(\frac{D g^{3}}{27 \rho}\right)^{1 / 2}
$$

the critical speed originally identified by Kheysin [2] that is equivalent to $c_{\min }$.

As previously indicated, the singularity in the solution (9) at the critical load speed $V=c_{\min }$ corresponds to a continuously growing response, when the implicit steady-state assumption is not valid. We can always determine the relevant asymptotic time-dependent response (as $t \rightarrow \infty$ ) for any load speed, directly from (8). Thus on first exchanging the order of the integration and then introducing a familiar asymptotic form for the Bessel function $J_{0}(k V s)$, we get

$$
\begin{aligned}
\zeta(t) \approx & \zeta(T)-\frac{P_{0}}{\sqrt{2 \pi^{3} V} R} \int_{T}^{t} \frac{1}{\sqrt{s}} \int_{0}^{\infty} \frac{\tanh (k H) J_{1}(k R)}{\sqrt{k} c(k)} \\
& \times\left(\sin \left[k(c-V) s+\frac{\pi}{4}\right]+\sin \left[k(c+V) s-\frac{\pi}{4}\right]\right) d k d s
\end{aligned}
$$

for sufficiently large fixed $T>0$. Consequently, we may consider the stationary points for the potentially asymptotically-dominant integral (for $s \rightarrow \infty$ )

$$
I(s)=\operatorname{Im}\left(\int_{0}^{\infty} \frac{\tanh (k H) J_{1}(k R)}{\sqrt{k} c(k)} e^{i k(c-v) s} d k\right)
$$

where $\operatorname{Im}(z)$ denotes the imaginary part of $z$, especially with reference to the existence of any response that does not tend to a steady state. 
Thus when $V \rightarrow \sqrt{g H}$ from below, we have integral (11) represented asymptotically by

$$
\int_{0}^{\epsilon} \frac{H R}{2 V} k^{3 / 2} e^{-i H^{2} V k^{3} s / 6} d k=O\left(s^{-5 / 6}\right)
$$

as $s \rightarrow \infty$; and hence from (10) there is a transient

$$
O\left(\int_{T}^{t} \frac{1}{\sqrt{s}} s^{-5 / 6} d s\right)=O\left(t^{-1 / 3}\right)
$$

as $t \rightarrow \infty$, so the deflexion tends to a steady state. In the neighbourhood of $k=k_{\min }$ however, a similar asymptotic evaluation of integral (11) produces a result $O\left(s^{-1 / 2}\right)$, so that as $V \rightarrow c_{\min }$ from above the deflexion grows as

$$
O\left(\int_{T}^{t} \frac{1}{\sqrt{s}} s^{-1 / 2} d s\right)=O(\ln t)
$$

A similar analysis based upon (7) can be made for the concentrated point load, serving to emphasise that load concentration not only does not affect the time-dependence of the deflexion underneath the centre of the load, but also that this time-dependence should be seen at any field position where the disturbance is measurable.

Thus we conclude that the two-dimensional time-dependent analysis predicts that the deflexion at the load speed $V=\sqrt{g H}$ approaches a steady state, but at the critical load speed $V=c_{\min }$ the deflexion continues to grow with time, consistent with the calculations made by Milinazzo et al. [5] for a steadily-moving distributed load. This is in contrast with the prediction from one-dimensional time-dependent analysis for a line load $([3,8])$, that the deflexion grows with time at both of these speeds (viz. as $t^{1 / 3}$ and $t^{1 / 2}$, respectively). Indeed, in our two-dimensional analysis the transient decay at other load speeds is enhanced by a factor $t^{-1 / 2}$ in comparison with the one-dimensional results for a line load, as one might expect when energy may radiate away in directions other than along the line of motion of the load.

\section{Summary}

Detailed asymptotic and numerical analysis allows us to interpret formal Fourier integral solutions for the deflexion produced by a load moving over a continuouslysupported flexible plate. For a steady load travelling with uniform velocity, we can determine the distinctive wave patterns that depend upon the load speed. Analysis of the time-dependent Fourier integral solution for the two-dimensional response due to an impulsively-started concentrated point load, or for the response underneath a distributed load, demonstrates that a steady state is achieved at all load speeds except 
the critical load speed coincident with the minimum phase speed of the generated hybrid waves, when the deflexion grows continuously with time (in the absence of dissipation).

\section{References}

[1] J. W. Davys, R. J. Hosking and A. D. Sneyd, "Waves due to a steadily moving source on a floating ice plate", J. Fluid Mech. 159 (1985) 269-287.

[2] D. Ye. Kheysin, "Moving load on an elastic plate which floats on the surface of an ideal fluid", Izvestiya Akademii Nauk S.S.S.R., Otd. Tekh. Nauk, Mekhanika i Mashinostroenie 1 (1963) 178180, (in Russian).

[3] D. Ye. Kheysin, "Some unsteady-state problems in ice-cover dynamics", in Studies in Ice Physics and Ice Engineering (ed. G. N. Yakovlev), (Israel Program for Scientific Translations, 1973) 69-78.

[4] J. Lighthill, Waves in Fluids (Cambridge University Press, 1978).

[5] F. Milinazzo, M. Shinbrot and N. W. Evans, "A mathematical analysis of the steady response of floating ice to the uniform motion of a rectangular load", J. Fluid Mech. 287 (1995) 173-197.

[6] D. E. Nevel, “Moving loads on a floating ice sheet”, CRREL Research Report 261, Cold Regions Research and Engineering Laboratory, Hanover, New Hampshire, USA, 1970.

[7] W. S. Nugroho, K. Wang, R. J. Hosking and F. Milinazzo, "Time-dependent response of a floating flexible plate to an impulsively-started steadily moving load", J. Fluid Mech. 381 (1999) 337-355.

[8] R. M. S. M. Schulkes and A. D. Sneyd, "Time-dependent response of a floating ice sheet to a steadily moving load", J. Fluid Mech. 186 (1988) 25-46.

[9] A. D. Sneyd and R. J. Hosking, "Seepage flow through homogeneous soil into a row of drain pipes", J. Hydrology 30 (1976) 127-146.

[10] V. A. Squire, R. J. Hosking, A. D. Kerr and P. J. Langhorne, Moving Loads on Ice Plates (Kluwer Academic Publishers, 1996).

[11] K. Wang and R. J. Hosking, "Time-dependent response of a floating flexible plate to an impulsivelystarted steadily moving uniform circular load", Department of Mathematics and Statistics Preprint 96/7, James Cook University, Queensland, Australia, 1996. 